\title{
THEORETICAL STUDIES OF AN ATMOSPHERE AROUND SATURN'S RINGS
}

\author{
M. DENNEFELD
}

Service d'Aéronomie, Verrières-le-Buisson, France

\begin{abstract}
The subject of this paper is an attempt to predict what can be seen in the vicinity of Saturn through a UV photometer on board the Mariner-Jupiter-Saturn mission. We will restrict ourselves to the study of atomic hydrogen and $\mathrm{OH}$ and to $\mathrm{H}_{2} \mathrm{O}$ which can be the parent molecule and will consider only the particles ejected from the rings and from Titan.
\end{abstract}

Until recently, only a few experiments have been conducted to detect an atmosphere around the rings of Saturn and the results are controversial (e.g. Franklin and Cook, 1969; Feibelman, 1967).

The subject of this paper is an attempt to predict what can be seen in the vicinity of Saturn through a UV photometer on board the Mariner-Jupiter-Saturn mission. Therefore we will restrict ourselves to the study of atomic hydrogen and $\mathrm{OH}$ and to $\mathrm{H}_{2} \mathrm{O}$ which can be the parent molecule.

We can, a priori, expect three sources:

- the atmosphere of Saturn,

- the rings,

- the atmosphere of Titan.

In fact, the atmosphere of Saturn can be neglected owing to the great mass of the planet.

It appears very rapidly that the atoms or molecules ejected from the rings form an 'atmosphere' totally uncoupled from the 'atmosphere' formed by the atoms ejected from Titan. Therefore, the two problems will be treated separately.

First, we neglect the hypothetical existence of a magnetic field on Saturn and all the dynamical problems will be treated as two-bodies problems.

\section{Particles Ejected from the Rings}

\subsection{SOURCES}

The only parameters of the rings we need to know are the optical thickness, the temperature and composition of the surface, which are directly obtained from observations. We use $\tau=1$ for ring B and $\tau=0.5$ for ring A, surface temperature $T \simeq 80 \mathrm{~K}$ and we assume that the ring's particles are coated with pure water ice. We neglect rings other than $\mathbf{A}$ or $\mathbf{B}$.

Under these conditions, the sources are:

\subsubsection{Evaporation of Ice}

At $T=80 \mathrm{~K}$, the vapor pressure is $P=1.1 \times 10^{-22}$ torr which gives a yield $S$ of $1.0 \times$ $\times 10^{3} \mathrm{H}_{2} \mathrm{O}$ molecules $\mathrm{m}^{-2} \mathrm{~s}^{-1}$, with a sticking coefficient equal to 1 . 
The total production is then $1.36 \times 10^{20} \mathrm{H}_{2} \mathrm{O} \mathrm{s}^{-1}$, with a mean square velocity $\bar{v}$ of $330 \mathrm{~ms}^{-1}$. But it must be pointed out that this production rate can be increased by a factor of $4 \times 10^{6}$ if the surface temperature is $100 \mathrm{~K}$.

\subsubsection{Bombardment by Meteorites}

The density of meteorites in the vicinity of the rings is taken from Cook and Franklin (1970) and is equal to $1.2 \times 10^{-23} \mathrm{~g} \mathrm{~cm}^{-3}$ with a mean velocity perpendicular to the plane of the rings $\bar{v}=15 \mathrm{~km} \mathrm{~s}^{-1}$.

The effect of the bombardment depends on the structure of the ring's particles.

(a) If the surface is coated with ice-crystals, solid particles are ejected the mass of which can be evaluated from the results of Gault et al. (1963) on lunar material. The ejected mass is about 3700 times the incoming mass for the range of velocities we consider. The ejection is then of about $2 \times 10^{4} \mathrm{~kg} \mathrm{~s}^{-1}$. This production is insufficient by several orders of magnitude to supply the density of small particles required by the model of Franklin and Cook (1965).

(b) If the surface is coated with snow, we can assume that a fraction $\eta$ (here taken equal to $\frac{1}{3}$, as in Cook and Franklin (1970)) of the incoming energy is used to evaporate water. This gives a mass of water vapor equal to 70 times the incoming mass but only $2.7 \%$ of this vapor can escape the surface (owing to the depth of the hole produced by the impact).

This gives a total production of $3.7 \times 10^{26} \mathrm{H}_{2} \mathrm{O} \mathrm{s}^{-1}$. The velocity of the ejected molecules depends on the heating but is unlikely to be greater than $3 \mathrm{~km} \mathrm{~s}^{-1}$.

\subsubsection{Bombardment by the Solar Wind}

As pointed out by Harrison and Schoen (1967), the bombardment by the solar wind can be an important source of mass loss for the rings. To evaluate the ejection rate more precisely, we extrapolate the results used for metals by, e.g., Dienes and Vineyard (1957) or Pease (1960) to the water ice case. It is not possible to predict with certainty whether the ejected particles are $\mathrm{H}, \mathrm{OH}$ or $\mathrm{H}_{2} \mathrm{O}$. We assume that the actual production rate is directly proportional to the production rate calculated by assuming that $\mathrm{H}$ or $\mathrm{OH}$ alone are present in the crystal. More detailed calculations, taking into account the actual structure of ice, will be achieved very soon. Assuming that the displacement energy $E_{d}=36 \mathrm{eV}$ is very high (by the experimental law $E_{d}=7 E_{s}$ where $E_{s}$ is $5.16 \mathrm{eV}$, binding energy $\mathrm{H}-\mathrm{OH}$ ), we obtain a lower limit for the yield if the mechanism proposed is operating. The results are given in Table $I$, for impact of a proton or an alpha-particle on $\mathrm{H}$ or $\mathrm{OH}$, assuming a velocity of $400 \mathrm{~km} \mathrm{~s}^{-1}$ for the solar wind. The same calculation was made for ejection of $\mathrm{H}_{2} \mathrm{O}$ assuming that in this case $E_{s}=0.52 \mathrm{eV}$, the energy of sublimation of water ice.

These values have to be considered carefully because the theory is very elementary. Laboratory measurements are planed, in collaboration with the group of M. Maurette, Laboratory R. Bernas, Orsay, France. The calculations don't allow us to predict the velocity at ejection. We can only say that the kinetic energy is at most of the order of one or two times the energy of sublimation. We then obtain the following produc- 
TABLE I

\begin{tabular}{lcr} 
& \multicolumn{2}{c}{ Sputtering yields } \\
Target & \multicolumn{2}{c}{ Incoming particle } \\
& $\mathrm{H}^{+}$ & \multicolumn{1}{c}{$\mathrm{He}^{++}$} \\
& & \\
& & \\
$\mathrm{H}$ & $7.2 \times 10^{-3}$ & $4 \times 10^{-2}$ \\
$\mathrm{OH}$ & $1.4 \times 10^{-2}$ & $9.6 \times 10^{-2}$ \\
$\mathrm{H}_{2} \mathrm{O}$ & 0.18 & 1.13 \\
& &
\end{tabular}

tion, assuming the flux of solar wind particles is $2.2 \times 10^{6} \mathrm{H}^{+} \mathrm{cm}^{-2} \mathrm{~s}^{-1}$ and $8.8 \times$ $\times 10^{4} \mathrm{He}^{++} \mathrm{cm}^{-2} \mathrm{~s}^{-1}$ at $9.54 \mathrm{AU}$ :

$7.5 \times 10^{23} \mathrm{H} \mathrm{s}^{-1}$ and $3 \times 10^{24} \mathrm{OH} \mathrm{s}^{-1}$ if there is no water ejection,

or

$$
8.7 \times 10^{22} \mathrm{H} \mathrm{s}^{-1}, 3 \times 10^{23} \mathrm{OH} \mathrm{s}^{-1}, 5 \times 10^{25} \mathrm{H}_{2} \mathrm{O} \mathrm{s}^{-1} .
$$

More details can be found in Dennefeld (1973).

\subsubsection{Optical Erosion}

This mechanism is probably not important enough because of the very low momentum of the photons with respect to the thermal momentum of the atoms in the crystal (Dexter, 1964).

\subsubsection{Effect of the Interstellar Wind}

The neutral hydrogen atoms flowing in the heliosphere have a kinetic energy at impact on the rings of Saturn which is at most equal to $16.4 \mathrm{eV}$, considering a velocity of $8 \mathrm{~km} \mathrm{~s}^{-1}$ at infinity (with respect to the Sun) and a balance between radiation pressure and gravitational attraction of the Sun. The threshold for ejection is about $20 \mathrm{eV}$ and therefore the hydrogen atoms of the interstellar wind have no effect.

On the other hand, if $\mathrm{He}$ atoms are present in the interstellar wind with the same velocity at infinity of $8 \mathrm{~km} \mathrm{~s}^{-1}$, their energy at impact will be sufficient to eject $\mathrm{H}_{2} \mathrm{O}$ molecules, but not hydrogen or $\mathrm{OH}$.

Using the model of interstellar wind calculated by Ammar (1973) (density of $0.01 \mathrm{He} \mathrm{cm}^{-3}$ at infinity), we obtain a production rate of water between $4.4 \times$ $\times 10^{24} \mathrm{H}_{2} \mathrm{O} \mathrm{s}^{-1}$ and $1.3 \times 10^{24} \mathrm{H}_{2} \mathrm{O} \mathrm{s}^{-1}$, depending on the position of Saturn on his orbit. This production rate is ten times lower than the production rate by solar wind particles and will be preponderant only when the rings are seen edgewise from the Sun.

The production rates by all these mechanisms are summarized in Table II. The uncertainty can be as great as a factor of 2 or 3 .

\subsection{Distribution OF EJeCted Particles}

The orbit of each individual particle has been calculated in a two-bodies problem, assuming that all ejected particles have the same velocity and assuming a random distribution of the direction of ejection, in a reference frame bounded to the rings. 
TABLE II

Production of the particles

\begin{tabular}{|c|c|c|c|}
\hline Molecules & Mechanisms & Production rates & $\begin{array}{l}\text { Velocity } \\
\text { of ejected } \\
\text { particles }\end{array}$ \\
\hline $\mathrm{H}_{2} \mathrm{O}$ & $\begin{array}{l}\text { Evaporation of ice } \\
\text { Meteoroidal bombardment } \\
\text { Solar wind bombardment } \\
\text { Interstellar wind }(\mathrm{He}) \\
\text { bombardment }\end{array}$ & $\begin{array}{l}1.4 \times 10^{20} \mathrm{~s}^{-1} \\
3.7 \times 10^{26} \mathrm{~s}^{-1} \\
5.1 \times 10^{25} \mathrm{~s}^{-1} \\
4.4 \times 10^{24} \mathrm{~s}^{-1}\end{array}$ & $\begin{aligned} & 330 \mathrm{~ms}^{-1} \\
& 1 \mathrm{~km} \mathrm{~s}^{-1} \\
< & 3 \mathrm{~km} \mathrm{~s}^{-1} \\
< & 3 \mathrm{kms}^{-1}\end{aligned}$ \\
\hline $\mathbf{H}$ & Solar wind bombardment & $7.5 \times 10^{23} \mathrm{~s}^{-1}$ & $<44 \mathrm{kms}^{-1}$ \\
\hline $\mathrm{OH}$ & Solar wind bombardment & $3 \times 10^{24} \mathrm{~s}^{-1}$ & $<10 \mathrm{kms}^{-1}$ \\
\hline
\end{tabular}

We have the following results:

- As long as the ejection velocity $V_{i}$ is much lower than the circular velocity, the dependency of the characteristics of the orbit upon the distance of ejection is small.

- The loss rate by escape or falling into the planet is less than $5 \%$ if $V_{i}<3 \mathrm{~km} \mathrm{~s}^{-1}$ (Figure 1).
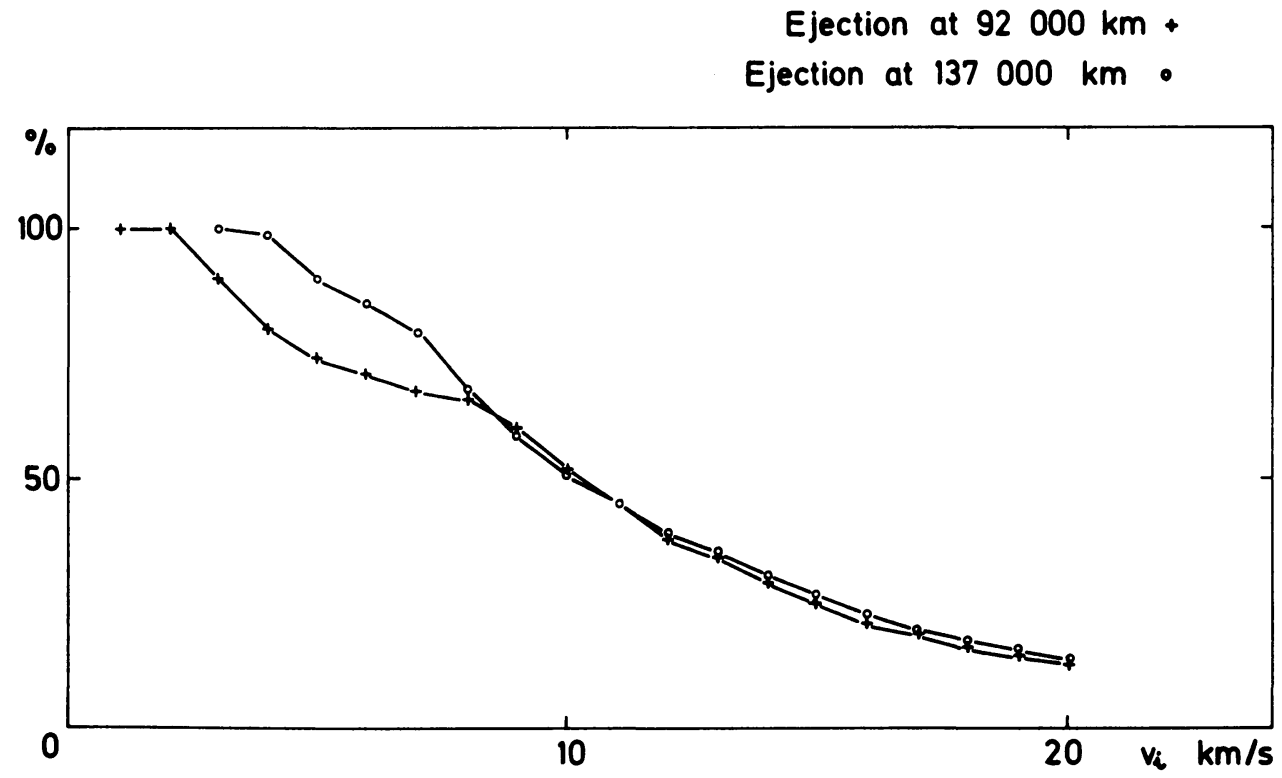

Fig. 1. Particles in 'stable' orbits around the rings. Abcissa: velocity of ejection with respect to the ring's particles. We assume that all particles have the same velocity. Ordinates: percentage of ejected particles.

If $V_{i}<3 \mathrm{~km} \mathrm{~s}^{-1}$, the volume occupied by the particles depends only on the ejection velocity and on the greatest distance of ejection. It has a toroidal form, the section of which can be seen in Figure 2. 
After one orbit, the atom or molecule collides with the rings and the probability of flowing through without capture is $\varepsilon=1-\tau S_{t}$ where $\tau$ is the optical thickness and $S_{t}$ the sticking coefficient. The total number of atoms in the volume is then $Q T /(1-\varepsilon)$, where $Q$ is the production rate, $T$ the mean orbital period and $(\varepsilon<1$.

The lifetime against.capture is then $T /(1-\varepsilon)$, to be compared with the lifetime against photodissociation by solar EUV photons.

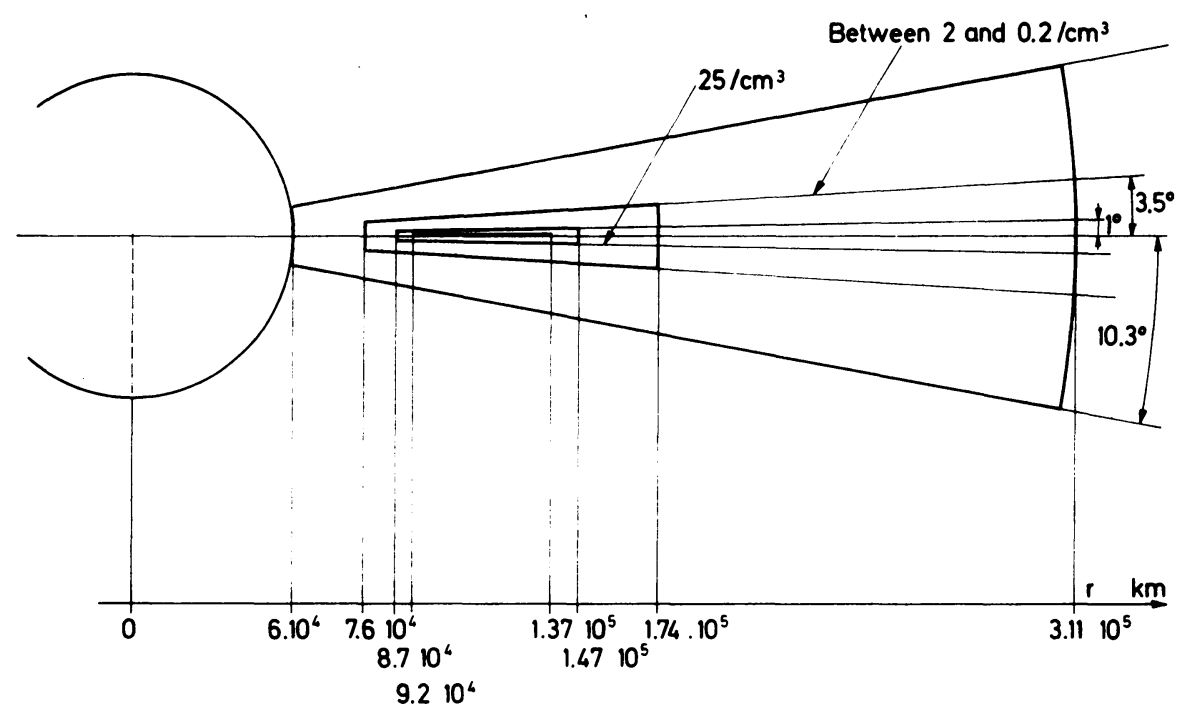

Fig. 2. Volume occupied by water vapor around the rings: section perpendicular to the plane of the rings. We distinguish between different origins of water.

\subsubsection{Case of $\mathrm{H}_{2} \mathrm{O}$ Molecules}

We take $S_{t}=0.5$ (e.g. Bruce, 1954) and $\tau=1$ everywhere. Then $\varepsilon=0.5, T /(1-\varepsilon)=$ $=2 T \simeq 8 \times 10^{4} \mathrm{~s}$. The lifetime against photodissociation is $9 \times 10^{6} \mathrm{~s}$ at $9.54 \mathrm{AU}$ for $\mathrm{H}_{2} \mathrm{O}$ (Massey et al., 1969; Hinteregger, 1970). We therefore deduce that water is not dissociated before capture by the rings. This means that the total loss of matter by the rings due to the ejection of water is very low, contrary to what has been claimed previously (e.g. Harrison and Schoen, 1967).

The densities obtained are the following, depending on the ejection mechanism which governs the velocity after ejection:
- Evaporation
$5 \times 10^{-5} \mathrm{H}_{2} \mathrm{O} \mathrm{cm} \mathrm{cm}^{-3}$
- Solar wind bombardment
0.18
$\mathrm{H}_{2} \mathrm{O} \mathrm{cm}^{-3}$
- Meteoroidal bombardment
24
$\mathrm{H}_{2} \mathrm{O} \mathrm{cm}^{-3}$
- Interstellar wind bombardment $\quad 0.02$
$\mathrm{H}_{2} \mathrm{O} \mathrm{cm} \mathrm{cm}^{-3}$.

All these volumes are shown in Figure 2.

Detection by $\mathbf{H} \mathrm{L} \alpha$ absorption is impossible: the optical depth is about $10^{-5}$ and detection in the infrared seems to be very difficult. 


\subsubsection{Case of $H$ Atoms}

The lifetime is limited by photoionization and charge exchange: $1.5 \times 10^{8} \mathrm{~s}$ at $9.54 \mathrm{AU}$ because the sticking coefficient in ice is very low, and therefore an $\mathrm{H}$ atom suffers many collisions before destruction. The volume occupied must then be spherical, with a radius of $3.1 \times 10^{5} \mathrm{~km}$ if the velocity at ejection is $3 \mathrm{~km} \mathrm{~s}^{-1}$. The density is then about $1 \mathrm{H} \mathrm{cm}^{-3}$, giving a brightness of 1.3 rayleigh, detectable by a $\mathrm{L} \alpha$ photometer. This density can be considerably reduced if the velocity at ejection is higher than $3 \mathrm{~km} \mathrm{~s}^{-1}$.

\subsubsection{Case of $\mathrm{OH}$ Radicals}

If the velocity at ejection is $3 \mathrm{~km} \mathrm{~s}^{-1}$, density becomes $90 \mathrm{O} \mathrm{cm}^{-3}$ in the same spherical volume than before. The brightness at $3090 \AA$ is then about 6.5 rayleigh. The same remark as for $\mathrm{H}$ is valid here.

\subsection{Conclusion}

We have pointed out the existence of toroïdal or spherical atmospheres around the rings of Saturn.

The observation of these atmospheres seems to give no very important results concerning the size of particles in the rings. The only directly observable parameter is the velocity at ejection which depends on the surface-state of the rings-particles in a manner which can be well known only after laboratory-experiments.

On the other hand, water density is very sensitive to surface temperature. The mass of the rings can be attained through perturbation of individual orbits only if it is higher than about $10^{-3}$ times the mass of Saturn, and this is very unlikely.

However, if there are Van Allen Belts around Saturn, the flux of particles colliding with the rings can be much higher. In this case, the brightness of the 'atmosphere' will be increased too and the flux of trapped particles can be deduced from the study of $\mathrm{H} \mathrm{L} \alpha$ emission around the rings.

\section{Atoms Ejected from Titan}

We can distinguish between escape of atomic hydrogen and escape of molecular hydrogen.

The escape flux depends strongly on the model used.

\subsection{ESCAPE OF ATOMIC HYDROGEN}

We shall try to set an upper limit to the escape flux of atomic hydrogen in the following manner: the parent molecules of atomic hydrogen can be $\mathrm{H}_{2}$ or $\mathrm{CH}_{4}$ in the atmosphere of Titan (we shall speak later about $\mathrm{NH}_{3}$ ). We shall assume that all the solar EUV flux able to destruct these parent molecules is absorbed with an efficiency of 1 . In this case, the flux to be used is that below $1450 \AA$. The input at $9.54 \mathrm{AU}$ is then $5.5 \times 10^{9}$ photons $\mathrm{cm}^{-2} \mathrm{~s}^{-1}$ (Hinteregger, 1970). Assuming that once a photon of 
$\lambda<1450 \AA$ penetrates below a radial distance of, say, $10000 \mathrm{~km}$, it produces a $\mathrm{H}$ atom, the total number of $\mathrm{H}$ atoms produced is then $1.5 \times 10^{28} \mathrm{~s}^{-1}$. We further assume that, in a steady state, each atom produced will escape. These atoms will have a Maxwellian distribution of velocities at an exospheric temperature of about $80 \mathrm{~K}$. To obtain an upper limit, we assume that the mean square velocity is equal to the escape velocity (limit of blow-off) at the critical level. Table III shows then that only a few percent of the atoms can escape from the Saturnian system, as long as the exospheric temperature is lower than $120 \mathrm{~K}$. See also Figure 3.

The volume occupied has a toroïdal form, the section of which is shown in Figure 4.

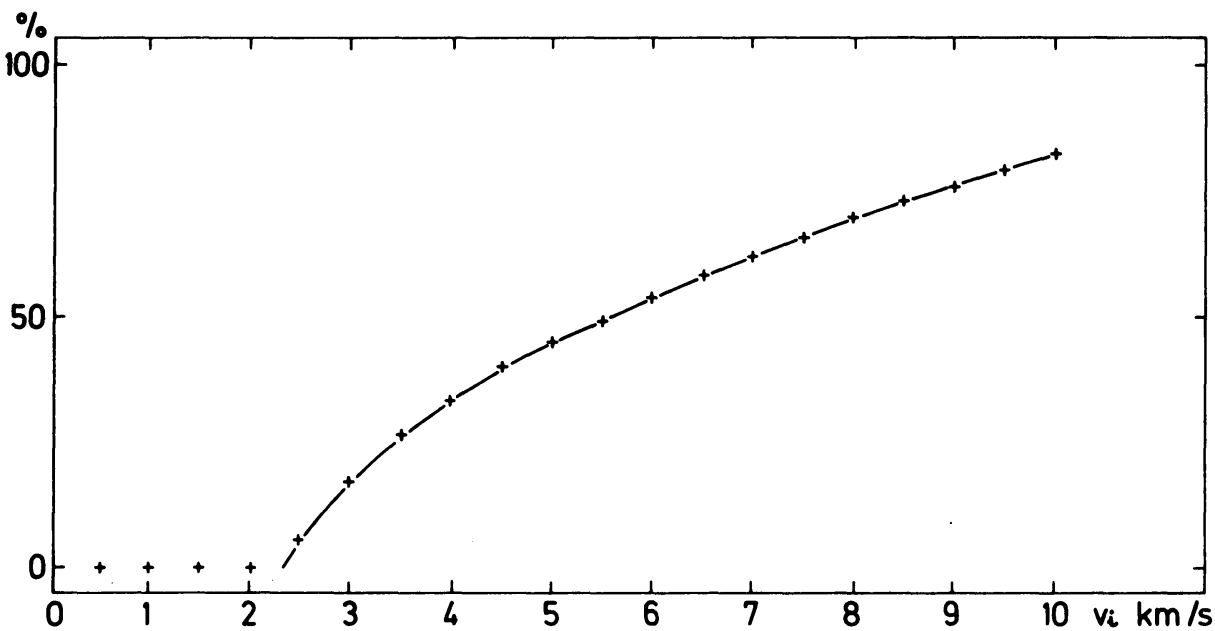

Fig. 3. Particles ejected from Titan which also escape from the system of Saturn. Abcissa: ejection velocity with respect to Titan. We assume here that all particles have the same velocity. Ordinates: percentage of ejected particles.

TABLE III

Escape of particles from the Saturnian system

\begin{tabular}{|c|c|c|c|c|c|}
\hline $\begin{array}{l}\text { Exo- } \\
\text { spheric } \\
\text { temperature }\end{array}$ & $\begin{array}{l}\% \text { of produced } \\
\text { particles with } \\
\text { escape velocities } \\
\text { from Titan }\end{array}$ & $\begin{array}{l}\% \text { of produced } \\
\text { particles with } \\
\text { velocities } \\
>2.3 \mathrm{~km} \mathrm{~s}^{-1}\end{array}$ & $\begin{array}{l}\text { Rates of } \\
\text { maximum } \\
\text { escape }\end{array}$ & $\begin{array}{l}\text { Escape from } \\
\text { the Saturn } \\
\text { system (in \% } \\
\text { of ejected } \\
\text { particles) }\end{array}$ & $\begin{array}{l}\text { Escape from } \\
\text { the Saturn } \\
\text { system (in \% of } \\
\text { particles escaped } \\
\text { from Titan) }\end{array}$ \\
\hline 70 & 39 & 2.8 & 6.0 & 0.17 & 0.44 \\
\hline 80 & 39 & 4.7 & 11.0 & 0.52 & 1.3 \\
\hline 90 & 39 & 7.0 & 16.0 & 1.1 & 2.9 \\
\hline 100 & 39 & 9.5 & 19.5 & 1.9 & 4.9 \\
\hline 110 & 39 & 12.3 & 22.0 & 2.7 & 6.9 \\
\hline 120 & 39 & 15.1 & 23.0 & 3.5 & 9.0 \\
\hline
\end{tabular}




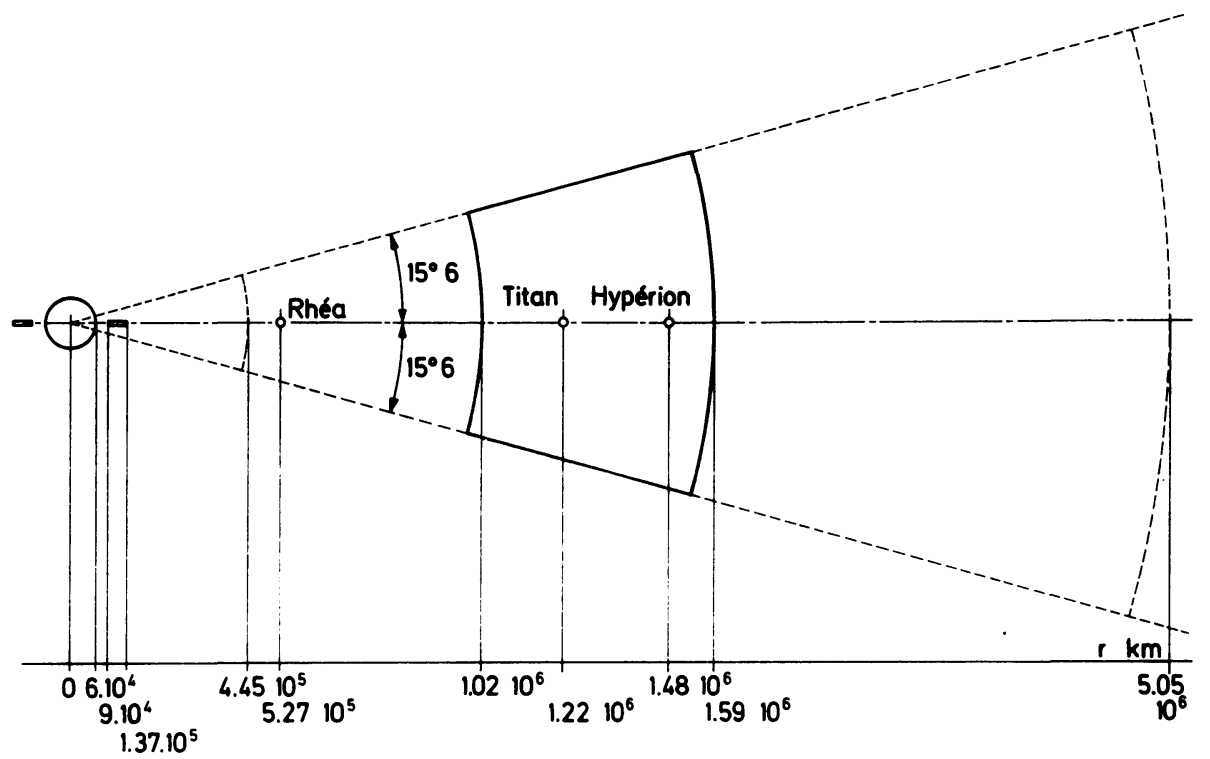

Fig. 4. Volume occupied by atomic hydrogen around the orbit of Titan: section perpendicular to the plane of orbit. The section in full lines has density 3 times greater than the mean density.

The mean density is given by:

$$
\bar{n}=\frac{Q}{V+S_{e} v_{\mathrm{T}}},
$$

where $Q$ is the production rate, $\tau$ the lifetime against ionization and charge exchange, $v_{\mathrm{T}}$ the orbital velocity of Titan and $S_{e}$ the surface of capture (taken as before equal to a sphere of radius $10000 \mathrm{~km}$ ).

With $Q=1.5 \times 10^{28} \mathrm{~s}^{-1}, V=1.5 \times 10^{29} \mathrm{~m}^{3}, \tau=1.5 \times 10^{8} \mathrm{~s}, S_{e}=3.14 \times 10^{14} \mathrm{~m}^{2}, v_{\mathrm{T}}=$ $=5.57 \mathrm{~km} \mathrm{~s}^{-1}$, the mean density becomes:

$$
\bar{n}=15 \mathrm{H} \mathrm{cm}^{-3} \text {. }
$$

The loss rate by capture by Titan (term $S_{e} v_{\mathrm{T}}$ ) is negligible with respect to the loss rate by photoionization or charge exchange.

In the volume shown in full lines in Figure 4 the density is about 3 times greater than the mean density. This atmosphere is optically thick in $L \alpha 1216 \AA$. The optical depth is about 4 along a line of sight lying in the plane of the rings. Assuming that we can see up to an optical depth of $\tau=1$, the brightness is then

$$
4 \pi B=95 \text { rayleigh. }
$$

\subsection{ESCAPE OF MOLECULAR HYDROGEN}

We use the model of Trafton (1972). The escape flux is $2 \times 10^{9} \mathrm{~cm}^{-2} \mathrm{~s}^{-1}$ for an 
exospheric temperature of $74 \mathrm{~K}$. This gives a production rate equal to the production rate of atomic hydrogen calculated before. Once ejected from Titan, the principal destruction process of molecular hydrogen is photoionization $\left(\sigma \sim 10^{-17} \mathrm{~cm}^{2}\right.$, $\lambda<804 \AA)$ rather than photo-dissociation $\left(\sigma \sim 10^{-18} \mathrm{~cm}^{2}, \lambda<840 \AA\right)$.

The contribution to the density of atomic hydrogen is therefore only $20 \%$ of that calculated previously.

But if this escape of molecular hydrogen is the dominant one (the escape of atomic hydrogen can be much lower than $1.5 \times 10^{28} \mathrm{~s}^{-1}$ if recombination in the atmosphere takes place) we have a mean density $3 \mathrm{H} \mathrm{cm}^{-3}$ and a brightness of $72 \mathrm{R}$. In this case, the 'atmosphere' is optically thin.

In all cases, we can conclude with the existence of an 'atmosphere' of atomic hydrogen around Titan.

More details can be found in Dennefeld (1973). Recently, Hunten (1973) published a model of Titan with an escape flux of the order of $10^{11} \mathrm{~cm}^{-2} \mathrm{~s}^{-1}$ at a critical level of $11000 \mathrm{~km}$. The total escape is then $1.5 \times 10^{30} \mathrm{H}_{2} \mathrm{~s}^{-1}$ which is 300 times greater than the value used by Trafton. With the same escape velocity as before, $1.4 \mathrm{~km} \mathrm{~s}^{-1}$, this gives a mean density of $1150 \mathrm{~cm}^{-3}$ (only $10 \%$ of the molecules are dissociated into $\mathbf{H}+\mathbf{H})$.

In this case, we have still a mean brightness of the order of $95 \mathrm{R}$, but the optical thickness at center is very high, of the order of 100 . A model using multiple diffusion of radiation must then be used to determine the actual brightness. With such a high density, each atom will suffer many collisions before destruction and we can infer from this that the volume will not be inevitably toroïdal, but can be spherical in shape, or flat, depending on the momentum-transfer in collisions.

The observation of the $\mathrm{L} \alpha$ airglow in the vicinity of the orbit of Titan can therefore give us the escape flux and an indication about the existence of $\mathrm{NH}_{3}$ in the atmosphere of Titan which seems to be the only candidate to supply such a high escape flux.

It must be pointed out that in the case of the presence of $\mathrm{NH}_{3}$ the upper limit of escape of atomic hydrogen will be increased by more than a factor of $100(\lambda<2400 \AA$, instead of $\lambda<1450 \AA$ ).

An other point is the existence in the vicinity of Titan of a flux of charged particles in the magnetosphere of Saturn. This has been studied by Brice and McDonough (1973). The density of atomic hydrogen can be reduced if the flux of charged particles is as high as the flux of the solar wind at $9.54 \mathrm{AU}, 2 \times 10^{6} \mathrm{H}^{+} \mathrm{cm}^{-2} \mathrm{~s}^{-1}$ which seems quite possible. In this case, the distribution in the toroidal volume will be asymmetric owing to the fact that the flux of charged particles decreases with radial distance to Saturn. Therefore it is possible to distinguish between a loss due to magnetospheric particles and a lower escape flux from Titan.

\subsection{Conclusion}

There are two independent 'atmospheres' in the system of Saturn: one around the rings, the other around the orbit of Titan.

- The 'atmosphere' around the rings contains water, the density of which can give 
us the surface state of the ring's particles: ice or snow. Predictions about $\mathrm{H}$ or $\mathrm{OH}$ are not easy as long as laboratory experiments are not made.

The existence and the flux of trapped particles in hypothetical Van Allen Belts are directly related to the brightness of this atmosphere.

- The 'atmosphere' around Titan contains $\mathbf{H}$ and $\mathbf{H}_{2}$, his size and extent depends on the escape flux from Titan.

If the form is spherical, the magnetic field of Saturn has no effect on the lifetime of $\mathrm{H}$ atoms at the distance of Titan and the escape flux is at least of the order of $5 \times 10^{10} \mathrm{~cm}^{-2} \mathrm{~s}^{-1}$. This possibly indicates the presence of $\mathrm{NH}_{3}$. A toroïdal form indicates a mean density low enough to make collisions negligible or an exchange of momentum in collisions which takes place preferentially perpendicularly to the plane of the orbit of Titan. The observation of the $\mathrm{L} \alpha$ glow gives us the escape flux and the exospheric temperature of Titan.

- The same calculations as before can be applied to the Galilean satellites of Jupiter, except Io, if we assume as a first step that they have no atmosphere. Assuming a surface temperature of the order of $130 \mathrm{~K}$, the water molecules evaporated (this is the dominant mechanism) from the surface (which is partially coated with ice, (Pilcher et al., 1972)) cannot escape the gravitational attraction of the body. They follow ballistic orbits before sticking on the surface. We have then a layer of water vapor on the surface, $75 \mathrm{~km}$ thick, with a density of the order of $5 \times 10^{7} \mathrm{~cm}^{-3}$, detectable by IR techniques. This number must be multiplied by a weighting factor less than one, which gives the proportion of the area coated with ice (Pilcher et al., 1972). This density is low enough to make flight collisions negligible. But, as suggested by Lewis, if ammonia-hydrates are present, the density can rise by a factor as high as $10^{5}$, leading to the existence of a real atmosphere. Full calculations will be published later.

\section{Acknowledgements}

I thank Drs J. Lewis and M. Maurette for helpful discussions. I thank Drs Brice and McDonough for kindly sending a preprint.

\section{References}

Ammar, A.: 1973, 3ème Cycle Thesis, to appear at University of Paris VI, France.

Brice, N. M. and McDonough, T. R.: 1973, Icarus 20, 136.

Bruce, E. W.: 1954, Trans. Instr. Chem. Engrs. London 32, 192.

Cook, A. F. and Franklin, F. A.: 1970, Icarus 75, 195.

Dennefeld, M.: 1973, 3ème Cycle Thesis University of Paris VI, France.

Dexter, D. L.: 1964, Nuovo Cimento 32, 90.

Dienes, G. J. and Vineyard, G. A.: 1957, in Radiation Effects in Solids, Interscience, New York, Ch. 3. Feibelman, W. A.: 1967, Nature 214, 793.

Franklin, F. A. and Cook, A. F.: 1965, Astron. J. 70, 704.

Franklin, F. A. and Cook, A. F.: 1969, Icarus 10, 417.

Gault, D. E., Shoemaker, E. M., and Moore, H. J.: 1963, NASA Techn. Note D-176.

Harrison, H. and Schoen, R. I.: 1967, Science 157, 1175.

Hinteregger, H. E.: 1970, Ann. Geophys. 26, 547. 
Hunten, D. M.: 1973, J. Atmospheric Sci. 30, 726.

Lewis, J. S.: 1971, Icarus 15, 174.

Massey, H. S. W., Burhop, E. H. S., and Gilbody, H. S.: 1969, in Electronic and Ionic Impact Phenomena, Clarendon Press, Oxford, Vol. II.

Pease, R. S.: 1960, Rendiconti S.I.F. 13, 158.

Pilcher, C. B., Ridgway, S. T., and McCord, T. R.: 1972, Science 178, 1087.

Trafton, L.: 1972, Astrophys. J. 175, 285. 